\title{
EFEITOS DA APLICAÇÃO DE REGULADORES VEGETAIS E DO ÁCIDO BÓRICO, EM ESTACAS DE LICHIEIRA (Litchi chinensis SONN.)
}

\author{
S. LEONEL \\ Dep. de Botânica do Instituto de Biociências/UNESP - CEP: 18618-000-Botucatu,SP. \\ J.D. RODRIGUES \\ Prof. Adjunto do Dep. de Botânica do Instituto de Biociências/UNESP - CEP: 18618-000-Botucatu,SP.
}

\begin{abstract}
RESUMO: Estudaram-se as interaçōes entre os ácidos indol-butírico, alfa-naftaleno-acético e bórico no desenvolvimento de calos e na sobrevivência das estacas de lichieira (Lüchi chinensis Sonn.). As estacas apresentavam $25 \mathrm{~cm}$ de comprimento e 4 folhas cortadas pela metade, sendo retiradas em duas épocas diferentes (janeiro e abril) e colocadas para enraizar em bandejas de isopor, tendo como substrato vermiculita e em condições de câmara de nebulização. Foi feita imersão de $2,5 \mathrm{~cm}$ da base das estacas, em soluçōes aquosas por 1 minuto. Os tratamentos utilizados corresponderam a 5.000 e $2.000 \mathrm{ppm}$ de IBA; 3.000 e $1.500 \mathrm{ppm}$ de $\mathrm{NAA} ; 150 \mathrm{microgramas} / \mathrm{ml}_{\mathrm{I}} \mathrm{de} \mathrm{H}_{3} \mathrm{BO}_{3}$; IBA 5.000 e $2.000 \mathrm{ppm}+\mathrm{H}_{3} \mathrm{BO}_{3} 150 \mathrm{microgramas} / \mathrm{ml}$; NAA 3.000 e $1.500 \mathrm{ppm}+\mathrm{H}_{3} \mathrm{BO}_{3} 150 \mathrm{microgramas} / \mathrm{ml}$ e $\mathrm{H}_{2} \mathrm{O}$. Avaliou-se a formação de calos e a sobrevivência das estacas após 120 dias do plantio. Através dos resultados obtidos, foi possivel concluir que dos tratamentos utilizados, nenhum foi efetivo na formação de raíres, havendo somente a formação de calos. A melhor época para a retirada de estacas correspondeu ao mês de abril.
\end{abstract}

Descritores: ácido indol-butírico, ácido naftaleno-acético, propagaçāo vegetativa.

\section{EFFECTS OF GROWTII REGULATORS AND BORIC ACID ON LYCITEE (Litchi chinensis SONN.) CUTTINGS}

\begin{abstract}
Interactions between indolebutyric, naphtalen acetic and boric acids were studied on "callus" development and survival in lychee (Lilchi chinensis Sonn.) cuttings. The cuttings were twenty five cm long with four leaves cut in half and taken in two different periods (January and April) Styrofoam trays, with vermiculite as substratum kept under intermittent mist were used as rooting media. Cuttings were imersed up to $2.5 \mathrm{~cm}$ from the base in water solutions, for one minute. The treatments were: 5,000 and 2,000 ppm of IBA; 3,000 and 1,500 ppm of NAA; 150 micrograms/ml $\mathrm{II}_{3} \mathrm{BO}_{3}$; $\mathrm{IBA} 5,000$ and $2,000 \mathrm{ppm}$ plus $\mathrm{H}_{3} \mathrm{BO}_{3} 150$ micrograms/ml; $\mathrm{NAA} 3,000$ and $1,500 \mathrm{ppm}$ plus boron 150 micrograms $/ \mathrm{ml}$ and $\mathrm{II}_{2} \mathrm{O}$. "Callus" formation and cutting survival were evaluated 120 days after planting. It was concluded that no treatments were effective on root formation, however, "callus" formation was observed. The best period for taking the cuttings was April.
\end{abstract}

Key Words: indolebutyric acid, naphtalen acetic acid, vegetative propagation.

\section{INTRODUÇÃO}

A lichia é uma fruta exótica, de origem chinesa, que está se tornando bastante conhecida no Brasil, principalmente no Estado de São Paulo, onde vem sendo muito procurada, atingindo excelentes preços para o produtor. No entanto, a expansão do seu cultivo se restringe, principalmente, devido à sua produção muito tardia, 10 a 15 anos, em pomares de pés-francos (GOMES, 1987). Esse tempo pode ser reduzido pela propagação vegetativa, a qual segundo JANICK (1966), elimina a fase juvenil da planta, tornando precoce a produção.
Tradicionalmente, a lichieira vem sendo propagada com eficiência através da alporquia; entretanto, tal processo acarreta, além de outros problemas, um alto custo para a produção das mudas (CHEN, 1949; CHANDLER, 1958; HARTMANN \& KESTER, 1983).

Ultimamente, têm sido intensificados os estudos sobre estaquia em fruticultura, os quais visam basicamente evitar os inconvenientes das variações genéticas, além de reduzir o longo período improdutivo.

De acordo com MENZEL (1985), a propagação de lichia, através de estacas, não é muito praticada por viveiristas devido às 
dificuldades apresentadas. $O$ sucesso da velocidade de multiplicação dessa planta depende de vários fatores, incluindo genótipos, condições fisiológicas da planta-mãe e condições ambientais.

Autores como JANICK (1966),
WEAVER (1982) e HARTMANN \& KESTER (1983), reportam que para um bom enraizamento $e$ necessária a presença nas estacas, de certo número de cofatores, que em combinação com as auxinas permitem que as estacas emitam raízes, sendo que a fonte desses cofatores estaria nas folhas. Para acelerar e promover o enraizamento de estacas, os mesmos autores recomendam o uso de reguladores de crescimento, mais especificamente, os ácidos indol-butírico (IBA) e alfa-naftaleno-acético (NAA). Além do fornecimento de auxinas exógenas, BRENCHLEY \& WARINGTON (1927) foram os primeiros pesquisadores que demonstraram a necessidade do boro para o desenvolvimento e crescimento das raízes adventícias, sendo mais tarde confirmados por REHM (1937), EATON (1940), JARVIS et al. (1983) e ONO (1990).

No presente trabalho, pretendeu-se estudar o comportamento de estacas de lichieira com a aplicação de fitorreguladores e ácido bórico, em diferentes épocas do ano.

\section{MATERIAL E MÉTODOS}

O experimento foi realizado em câmara de nebulização, sob ripado. As estacas foram retiradas de ramos terminais de lichieira com 19 anos de idade, pertencentes ao pomar da Fazenda Experimental Lageado e padronizadas de modo a apresentarem um comprimento de $25 \mathrm{~cm}$ (RAM \& MAJUMDAR, 1983), sendo mantidas 4 folhas cortadas pela metade, por estaca.

A época para retirada das estacas das plantas matrizes, correspondeu aos meses de janeiro e abril de 1989. Para cada mês de instalação foram utilizadas 360 estacas de lichieira, devidamente identificadas e distribuídas aleatoriamente, sendo cada tratamento constituído por 9 estacas por parcela, com 4 repetições, num delineamento inteiramente casualizado, onde os resultados foram submetidos à análise de variância (teste F), sendo as médias comparadas através do teste Tukey. A seguir, as estacas foram mergulhadas durante 1 minuto e a cerca de $2,5 \mathrm{~cm}$ da base em soluções que corresponderam aos tratamentos: IBA $5.000 \mathrm{ppm}$; IBA $2.000 \mathrm{ppm}$; IBA $5.000 \mathrm{ppm}+\mathrm{H}_{3} \mathrm{BO}_{3} 150$ microgramas $/ \mathrm{ml}$; IBA
$2.000 \mathrm{ppm}+\mathrm{H}_{3} \mathrm{BO}_{3} 150$ microgramas $/ \mathrm{ml}$; NAA $3.000 \mathrm{ppm}$; NAA $1.500 \mathrm{ppm}$; NAA $3.000 \mathrm{ppm}+$ $\mathrm{H}_{3} \mathrm{BO}_{3} 150$ microgramas/ml; NAA $1.500 \mathrm{ppm}+$ $\mathrm{H}_{3} \mathrm{BO}_{3} 150$ microgramas $/ \mathrm{ml} ; \mathrm{H}_{3} \mathrm{BO}_{3} 150$ microgramas $/ \mathrm{ml}$ e $\mathrm{H}_{2} \mathrm{O}$. $\mathrm{O}$ ácido alfa-naftalenoacético foi utilizado na forma do produto comercial NAFUSAKU, contendo $20 \%$ de NAA. Posteriormente, as estacas foram colocadas para enraizar em bandejas de isopor (68 x $34 \times 12 \mathrm{~cm}$ ) tendo, como substrato, vermiculita de granulação média e mantidas sob nebulização intermitente de 12 segundos, com intervalos regulares de 4 minutos.

\section{RESULTADOS E DISCUSSÃO}

A escolha da melhor época para a retirada das estacas das plantas matrizes, nem sempre é uma tarefa fácil e por isso tem sido preocupação de diversos pesquisadores, para diferentes espécies. No caso específico da lichieira, LENKA \& DAS (1981), coletando estacas dessa espécie durante seis meses, verificaram que somente as estacas coletadas em abril ou setembro (hemisfério sul) enraizaram, sendo que a maior porcentagem de enraizamento $(32,8 \%)$ foi obtida com as estacas coletadas em abril. RAM \& MAJUMDAR (1983), anelaram lotes de plantas de lichia no mês de junho (hemisfério sul), deixandoas uma semana para formação dos calos. Ainda sobre a mesma técnica, BOSE et al. (1985), anelaram estacas de ponteiro de lichia no mês de agosto (hemisfério sul), retirando-as depois de 15 dias.

Utilizando estacas de figueira (Ficus carica L.), PEREIRA et al. (1973) concluíram que a formação de raízes em estacas dessa espécie diminui com o decorrer da época de estaquia, sendo os meses de maio e junho aqueles onde a porcentagem de enraizamento é maior. SILVA et al. (1986), enraizaram estacas de diferentes porta-enxertos de videira, no período de julho a outubro, obtendo resultados diferentes para os pora-enxertos utilizados.

Este experimento foi montado em duas diferentes épocas do ano, correspondentes aos meses de janeiro e abril de 1989 , sendo que não foi obtido enraizamento das estacas em nenhuma época, havendo somente a formação de calos, nas estacas retiradas das plantas matrizes no mês de abril. A Figura 1 e a TABELA 1, mostram a porcentagem de sobrevivência das estacas de lichieira, aos 120 dias após a instalação no mês de janeiro. Através dos resultados apresentados, pode 


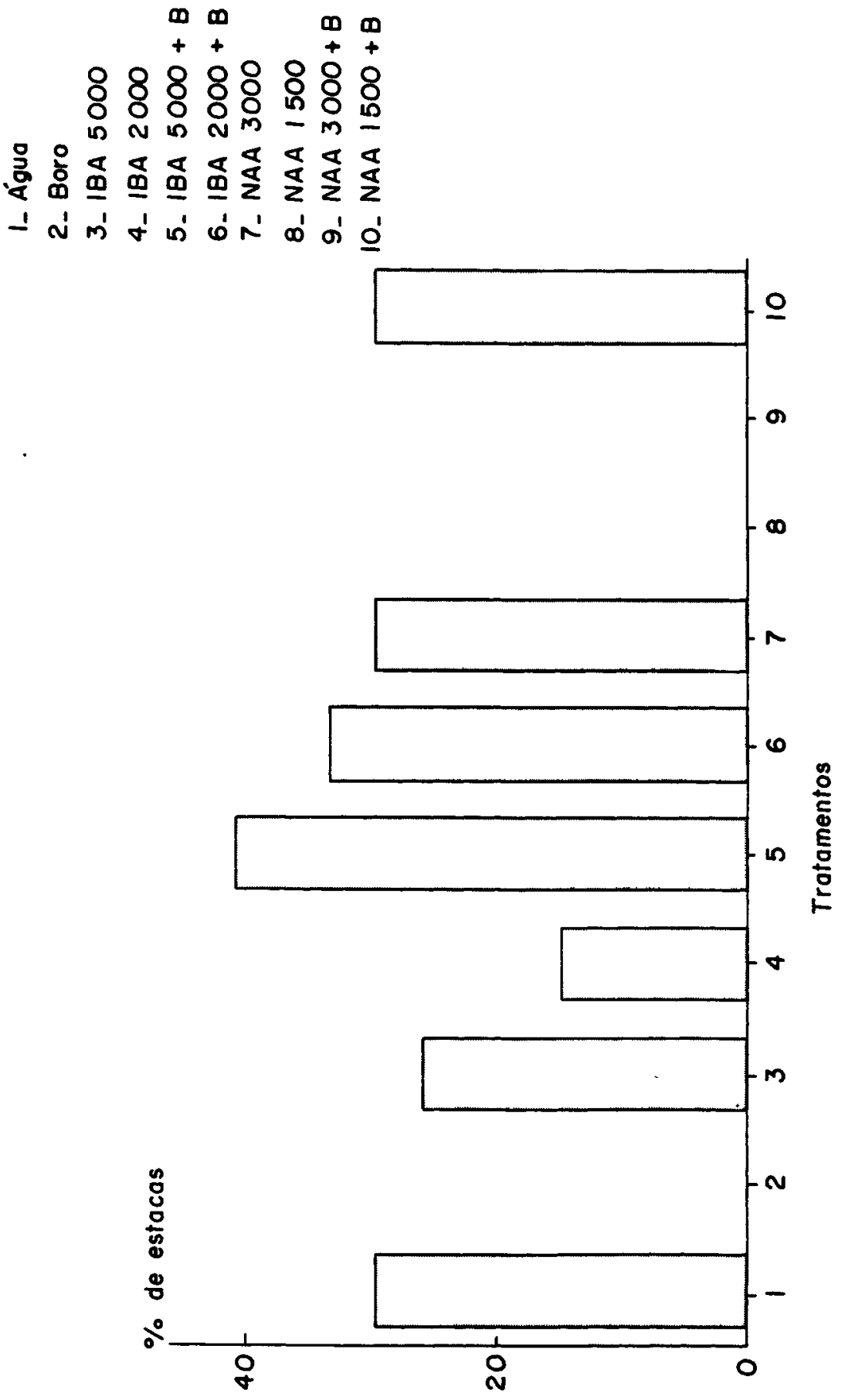



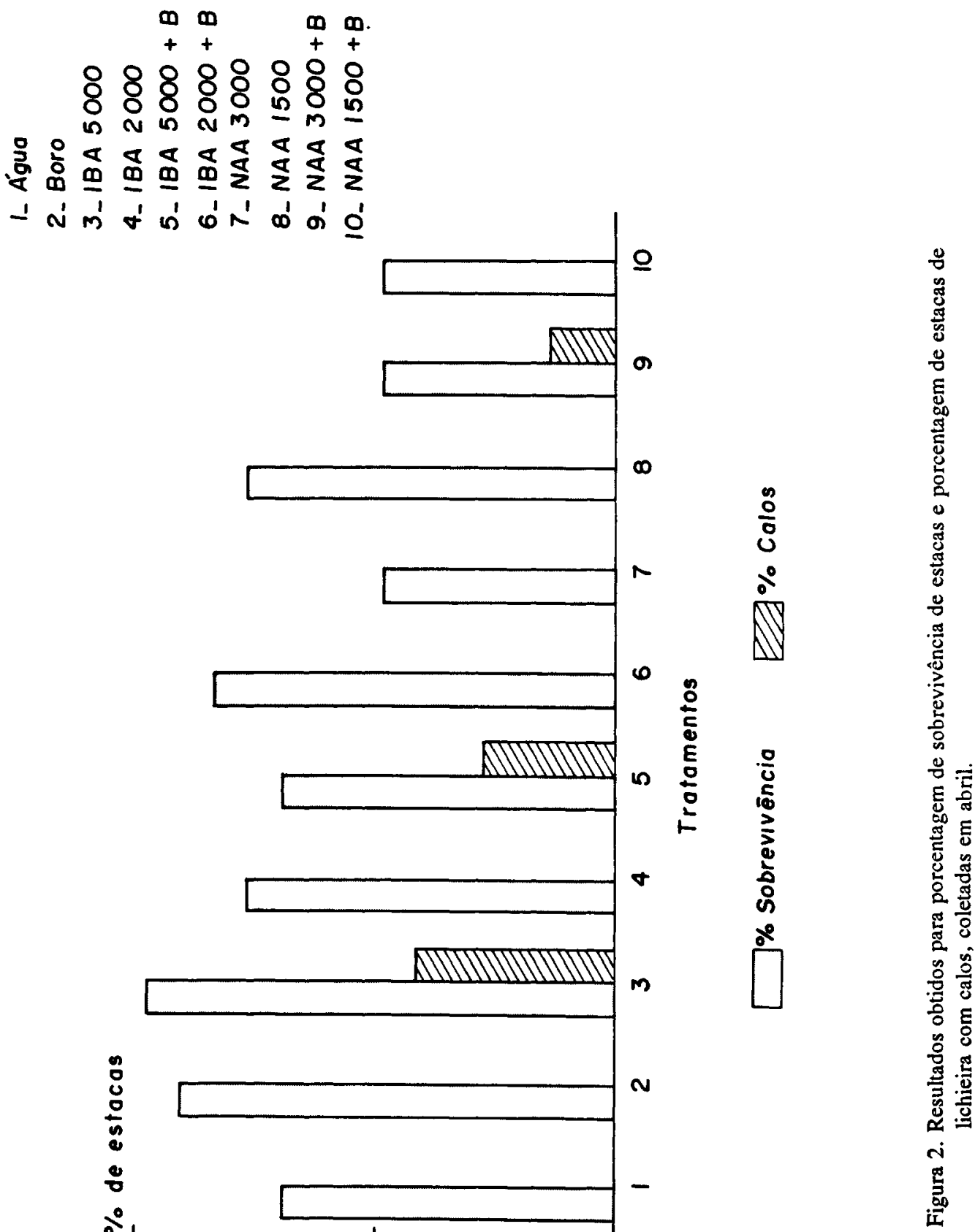
TABELA 1 - Resultados obtidos para a porcentagem de sobrevivência de estacas de lichieira nos meses de janeiro e abril, e porcentagem de estacas com calos no mês de abril.

\begin{tabular}{cccc}
\hline \hline Tratamentos & $\begin{array}{c}\text { \% Sobrevivência } \\
\text { Janeiro }\end{array}$ & $\begin{array}{c}\text { \% Sobrevivência } \\
\text { Abril }\end{array}$ & $\begin{array}{c}\text { \% Estacas com calos } \\
\text { Abril }\end{array}$ \\
\hline T1 & $29,63 \mathrm{ab}$ & $59,00 \mathrm{ab}$ & $0,00 \mathrm{~b}$ \\
T2 & $0,00 \mathrm{~b}$ & $77,00 \mathrm{a}$ & $0,00 \mathrm{~b}$ \\
T3 & $25,93 \mathrm{ab}$ & $83,00 \mathrm{a}$ & $33,33 \mathrm{a}$ \\
T4 & $14,81 \mathrm{ab}$ & $65,00 \mathrm{ab}$ & $0,00 \mathrm{~b}$ \\
T5 & $40,74 \mathrm{a}$ & $59,00 \mathrm{ab}$ & $22,20 \mathrm{ab}$ \\
T6 & $33,33 \mathrm{ab}$ & $71,00 \mathrm{a}$ & $0,00 \mathrm{~b}$ \\
T7 & $29,63 \mathrm{ab}$ & $41,00 \mathrm{~b}$ & $0,00 \mathrm{~b}$ \\
T8 & $0,00 \mathrm{~b}$ & $65,00 \mathrm{ab}$ & $0,00 \mathrm{~b}$ \\
T9 & $0,00 \mathrm{~b}$ & $41,00 \mathrm{~b}$ & $11,11 \mathrm{ab}$ \\
T10 & $29,63 \mathrm{ab}$ & $41,00 \mathrm{~b}$ & $0,00 \mathrm{~b}$ \\
\hline CV & $23,20 \%$ & $19,18 \%$ & $25,26 \%$ \\
F & $5,56 * *$ & $3,53 *$ & $4,98 *$ \\
\hline \hline
\end{tabular}

* $5 \%$ de nível de significância

** $1 \%$ de nível de significância

Mćdias seguidas da mesma letra não diferem entre si.

observar-se a baixa porcentagem de estacas que permaneceram vivas neste período. Os tratamentos com $\mathrm{H}_{3} \mathrm{BO}_{3} 150$ microgramas $/ \mathrm{ml}$, NAA $1.500 \mathrm{ppm}$ e NAA $3.000 \mathrm{ppm}+\mathrm{H}_{3} \mathrm{BO}_{3} 150$ microgramas $/ \mathrm{ml}$ resultaram em $100 \%$ de estacas mortas.

Esses resultados, de baixa porcentagem de sobrevivência, podem ser atribuídos à época inadequada para a estaquia, à inadequação do binômio concentração/tempo de imersão e também, às altas temperaturas verificadas nesta época do ano, sendo ainda mais elevadas no interior da câmara de nebulização. A variação na capacidade de enraizamento é atribuída às fases de crescimento da planta (HARTMANN \& LORETTI, 1965) e ao estado bioquímico das estacas (ONO, 1990).

A influência da temperatura do ar sobre o enraizamento das estacas já foi descrita. Assim, vários pesquisadores encontraram ser $24^{\circ} \mathrm{C}$ a temperatura mínima para o enraizamento e a ́́tima, para a maioria das variedades, está entre 27 e $32^{\circ} \mathrm{C}$
(HALMA, 1931; FORD, 1957; RYAN et al., 1958).

As altas temperaturas verificadas neste período de verão parecem exercer um efeito contrário ao enraizamento e à sobrevivência das estacas, devido talvez, à sua grande perda de água através da transpiração, não sendo reposta em quantidades suficientes. Observou-se, inicialmente, uma intensa queda de folhas e, posteriormente, a morte das estacas.

Os resultados, correspondentes à instalação no mês de abril de 1989, encontram-se na TABELA 1 e Figura 2. Esta época mostrou-se mais favorável para a sobrevivência das estacas de lichieira, havendo inclusive, a formação de calos em alguns tratamentos. $O$ uso do ácido indol-butírico (IBA) $5.000 \mathrm{ppm}$ e do IBA 5.000 ppm $+\mathrm{H}_{3} \mathrm{BO}_{3} 150$ microgramas $/ \mathrm{ml}$, resultou na maior porcentagem de estacas com calos, 33,33\% e $22,20 \%$, respectivamente (Figura 2). 
A não efetivação do enraizamento, havendo somente a formação dos calos, também pode ser devida à Epoca inadequada ou ao tempo de imersão das estacas, considerado impróprio para alguns tratamentos.

A escolha da combinação concentração/ tempo de imersão não $\varepsilon$ simples, uma vez que, concentrações pouco abaixo do nível crítico requerido para o enraizamento não são eficazes $e$, aquelas muito acima, tornam-se frequentemente fitotóxicas (WEAVER, 1982).

Durante este período, observou-se uma maior persistência das folhas nas estacas, fato este que pode ter favorecido uma maior sobrevivência e até a formação dos calos.

Assim é que, estudando o efeito do IBA na porcentagem de pegamento de estacas semi-lenhosas de pessegueiro, FACHINELLO \& KERSTEN (1981) analisaram os tratamentos 0 , $400,800,1.200$ e $1.600 \mathrm{ppm}$, aplicados por imersão durante 5 segundos na base das estacas (dois tipos, com duas folhas e sem folhas), concluindo não ter o IBA apresentado efeito nas estacas que não possuíam folhas.

Em estudos sobre os efeitos dos reguladores de crescimento, no enraizamento de estacas dos limoeiros Kagsi-Kalan, Baramasi e Eureka, com e sem folhas, ARORA \& YAMDAGNI (1985) observaram que é possível obter-se o enraizamento e a sobrevivência das estacas, independentemente da presença de folhas.

No entanto, foi necessário o uso de concentrações maiores dos fitorreguladores, nas estacas que não tinham folhas, para alcançar os objetivos propostos.

No Oeste de Bengala (Índia), POI \& MAZUMDAR (1989) realizaram experimento com estacas de Kath Bael (Limonia acidissima) com e sem folhas, sendo reportado pelos autores que somente aquelas que continham folhas enraizaram.

O efeito sinergístico do ácido bórico, no enraizamento de estacas, foi discutido por JARVIS et al. (1983), no qual o autor sugere que o boro possa regular níveis de auxinas endógenas, durante o desenvolvimento das raízes.

Para a instalação do mês de janeiro, o boro não teve papel no desenvolvimento de raízes, contudo, os tratamentos IBA $5.000 \mathrm{ppm}+\mathrm{H}_{3} \mathrm{BO}_{3}$ 150 microgramas e IBA $2.000 \mathrm{ppm}+\mathrm{H}_{3} \mathrm{BO}_{3} 150$ microgramas $/ \mathrm{ml}$, proporcionaram a maior sobrevivência das estacas $(40,74$ e $33,33 \%$, respectivamente).

\section{CONCLUSÕES}

- A persistência das folhas nas estacas possibilitou uma maior sobrevivência, estimulando a formação dos calos;

- O IBA foi mais eficiente que o NAA na porcentagem de sobrevivência e na formação dos calos, nas duas épocas de instalação do experimento (janeiro e abril);

- Foi observada a formação de calos somente nas estacas retiradas no mês de abril. A maior porcentagem de estacas com calos foi obtida com o IBA $5.000 \mathrm{ppm}(33,33 \%)$.

- O ácido bórico, aplicado isoladamente, não exerceu efeito favorável sobre a sobrevivência e a formação de calos nas estacas.

\section{REFERÊNCIAS BIBLIOGRÁFICAS}

ARORA, R.K.; YAMDAGNI, R. Effect of growth regulators on rooting of lemon cuttings with and without leaves. Haryana Agricultural University. Journal of the Research, Haryana, v.15, n.1, p.77-81, 1985 .

BOSE, T.K.; DHUA, R.S.; MITRA, S.K. et al. Synergism of phenolic substances with IBA in regeneration of root from litchi cuttings. Indian Journal of Horticulture, Bangalore, v.42, p.153-155, 1985.

BRENCHLEY, W.E.; WARINGTON, K. The role of boron in the growth of plants. Annual Botanical, London, v.41, p.167-187, 1927.

CHANDLER, W.H. Evergreen Orchards. Philadelphia: Lea \& Febiger, 1958. 452p.

CHEN, W.H. The culture of the lychee. Proceedings of the Florida State Horticultural Society, Winter Haven, v.62, p.223-226, 1949.

EATON, F.M. Interrelations in the effects of boron and indoleacetic acid on plant growth. Botanical Gazette, Chicago, v.101, p.700-705, 1940.

FACHINELLO, J.C.; KERSTEN, E. Efeito do ácido indol-butírico na porcentagem de estacas semi-lenhosas enraizadas de pessegueiro (Prunus persica (L.) Batsch), cv Diamante, em condições de nebulização. Revista Brasileira de Fruticultura, Cruz das Almas, v.3, p.49-50, 1981. 
FORD, H.W. A method of propagation citrus rootstocks clones by leaf bud cuttings. Proceedings of the American Society for Iorticultural Science, Alexandria, v.69, p.204-207, 1957.

GOMES, P. Fruticultura brasileira. 2.ed. São Paulo : Nobel, 1987. 448p.

HALMA, F.F. The propagation of citrus by cuttings. IIligardia, Oakland, v.6, n.5, p.131-157, 1931.

HARTMANN, H.T.; KESTER, D.E. Plant propagation: principles and practices. New York, Englewood Clipps, Prentice-Hall, 1983. 727p.

HARTMANN, H.T.; LORETTI, F. Seasonal variation in rooting leafy olive cuttings under mist. Proceedings ofthe Américan Society for Horticultural Science, Alexandria, v.87, p.194-198, 1965.

JANICK, J. A ciência da horticultura. Rio de Janeiro: USAID, 1966. 485p.

JARVIS, B.C.; ALI, A.H.N.; SHAHEED, A.I. Auxin and boron in relation to the response and ageing of ming bean cuttings. New Phytologist, Cambridge, v.95, p.509-518, 1983.

LENKA, P.C.; DAS, R.C. A note on the effect of IBA and rooting media on rooting of litchi stem cuttings under mist. Orissa Journal of IIorticultural, Orissa, v.9, n.1, p.40-41, 1981 .

MENZEL, C.M. Propagation of lychee: a review. Scientia IIorticulturae, Canterbury, v.25, p.31-48, 1985.

ONO, E.O. Interaçōes entre auxinas e ácido bórico no enraizamento de estacas caulinares de Coffea arabica L. cv "Mundo Novo". Botucatu, 1990. 162p. (Dissertação de Mestrado em Ciências Biológicas) Instituto de Biociências, Universidade Estadual Paulista "Julio de Mesquita Filho".
PEREIRA, F.M.; ABE, M.E.; JUNIOR, M.M.; et al. Influência da época de estaquia, em recipiente, no pegamento e desenvolvimento de estacas de figueira (Ficus carica L.). In: CONGRESSO BRASILEIRO DE FRUTICUlTURA, 1., 1971, Campinas. Anais... Campinas : Sociedade Brasileira de Fruticultura, 1973. p.446-450.

POI, A.K.; MAZUMDAR, B.C. Auxin induced root regeneration in stem cuttings of Kath Bael (Limonia acidissima). Indian Biologist, Calcutta, v.21, n.1, p.51-52, 1989.

RAM, M.; MAJUMDAR, D.K. Effect of indole butyric acid on stooling in lychee. Indian Journal of Ilorticulture, Bangalore, v.40, n.314, p.211-212, 1983.

REHM, S. Der einfluss der borsaure auf wachstum und saizaufhalme von Impatiens balsamina. Jb. wiss. Botanical, Berlin, v.85, n.788, 1937.

RYAN, G.F.; FROLICH, E.F.; KINSELLA, T.P. Some factors influencing rooting of grafted cuttings. Proceedings of the American Society for IIorticultural Science, Alexandria, v.72, p.454-461, 1958.

SILVA, A.L. da; FACHINELLO, I.C.; MACHADO, A.A. Efeito do ácido indolbutírico na enxertia e enraizamento da videira. Pesquisa Agropecuária Brasileira, Brasília, v.21, n.8, p.865-871, 1986.

WEAVER, R.J. Reguladores del crecimiento de las plantas en la agricultura. 2.ed. Barcelona : Trillas, 1982. 540p.

Trabalho entregue para publicação em 22.05 .92

Trabalho aprovado para publicação em 13.08.92 